\title{
Tamanho de amostra para a estimação da média de duração dos períodos larval, pupal e larval mais pupal de Microtheca spp. por bootstrap
}

\author{
Sample size for estimating the average duration of larval, pupal and larval stage plus pupal periods in \\ Microtheca spp. by bootstrap
}

\author{
Alberto Cargnelutti Filho ${ }^{I^{*}}$ Marcos Toebe $^{\mathrm{II}}$ Vinícius Soares Sturza $^{\mathrm{II}}$ Anderson Bolzan ${ }^{\mathrm{III}}$ \\ Tatiani Reis da Silveira ${ }^{\text {II }}$ Sônia Thereza Bastos Dequech ${ }^{\text {IV }}$
}

\section{- NOTA -}

RESUMO

O objetivo deste trabalho foi determinar o tamanho de amostra (número de insetos) para a estimação da média de duração dos períodos larval, pupal e larval + pupal de Microtheca ochroloma e de Microtheca semilaevis (Coleoptera: Chrysomelidae). Foram coletados adultos dessas espécies em uma área experimental de 0,25ha de nabo forrageiro (Raphanus sativus L.) e foi estabelecida uma criação em laboratório (temperatura de $25 \pm 2{ }^{\circ} \mathrm{C}$, umidade relativa de $60 \pm 10 \%$ e fotoperíodo de 12 horas). Após, foram mensurados os períodos larval, pupal e larval + pupal, em dias, de 119 e 81 insetos, respectivamente, de M. ochroloma e de M. semilaevis. Foram calculadas medidas de tendência central e de variabilidade. Foi determinado o tamanho de amostra por meio de bootstrap, com reposição de 10.000 amostras. Para a estimação da média dos períodos larval, pupal e larval + pupal, com intervalo de confiança de bootstrap de $95 \%$ de um dia, 42 e 35 insetos são suficientes, respectivamente, para $\mathbf{M}$. ochroloma e M. semilaevis.

Palavras-chave: Raphanus sativus L., besouros, reamostragem.

\section{ABSTRACT}

The objective of this research was to determine the sample size (number of insects) to estimate the average duration for larval, pupal and larval + pupal periods for M. ochroloma and M. semilaevis. Adults of both species were collected at a 0.25 ha experimental area of forage turnip (Raphanus sativus L.) and for laboratory rearing (temperature $25 \pm 2{ }^{\circ} \mathrm{C}$, relative humidity $60 \pm 10 \%$ and photoperiod 12 hours). Afterwards larval, pupal and larval + pupal periods were measured, in days, of 119 and 81 insects, respectively, M. ochroloma and $\boldsymbol{M}$. semilaevis. It was calculated measures of central tendency and variability and determined the sample size using bootstrap with replacement of 10000 samples. For estimating the average larval, pupal and larval + pupal periods, with amplitude of bootstrap confidence interval of $95 \%$, equal a day, 42 and 35 insects are sufficient, respectively for both M. ochroloma and M. semilaevis.

Key words: Raphanus sativus L., beetles, resampling.

As espécies Microtheca ochroloma Stal e Microtheca semilaevis Stal (Coleoptera: Chrysomelidae) são originárias da América do Sul (MANRIQUE et al., 2010) e, sendo insetos holometábolos, apresentam o ciclo biológico formado pelas fases de ovo, larva, pupa e adulto. Diversas espécies de brassicáceas, inclusive o nabo forrageiro (Raphanus sativus L.), são atacadas por larvas e por adultos desses besouros, que danificam os brotos terminais e as folhas (FARINHA et al., 2009).

'Departamento de Fitotecnia, Centro de Ciências Rurais (CCR), Universidade Federal de Santa Maria (UFSM), 97105-900, Santa Maria, RS, Brasil. E-mail: cargnelutti@pq.cnpq.br. *Autor para correspondência.

"Programa de Pós-graduação em Agronomia, UFSM, Santa Maria, RS, Brasil.

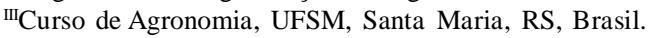

${ }^{\text {IV }}$ Departamento de Defesa Fitossanitária, CCR, UFSM, Santa Maria, RS, Brasil. 
É importante determinar, com precisão, a duração dos períodos larval, pupal e larval + pupal de M. ochroloma e de M. semilaevis. Essa informação é útil para estimar o tempo potencial em que os insetos podem estar presentes sobre as plantas e, também, a duração do ciclo biológico, o que possibilita estimar quantas gerações desta espécie podem se desenvolver durante o ciclo de uma cultura. Além disso, em estudos relacionados ao período de controle e à estimação do nível de dano, é importante conhecer a duração dessas fases.

Estudos sobre o dimensionamento do tamanho de amostra e do número de amostragens de larvas de Diloboderus abderus Sturm (SILVA \& COSTA, 1998) e sobre o número de plantas e de folhas de erva-mate que devem ser amostradas para o monitoramento do ácaro Dichopelmus notus Keifer (LÚCIO et al., 2009) têm sido realizados. No entanto, estudos que determinem o tamanho de amostra necessário para a estimação da média de duração dos períodos larval, pupal e larval + pupal de M. ochroloma e de M. semilaevis não foram encontrados na literatura. Assim, o objetivo deste trabalho foi determinar o tamanho de amostra (número de insetos) para a estimação da média de duração dos períodos larval, pupal e larval + pupal de $\boldsymbol{M}$. ochroloma e de $\boldsymbol{M}$. semilaevis.

Procedeu-se à semeadura a lanço da cultura de nabo forrageiro (Raphanus sativus L.) no dia 10/06/ 2010, em área experimental, de 0,25ha, do Departamento de Fitotecnia da Universidade Federal de Santa Maria (UFSM), Santa Maria, Estado do Rio Grande do Sul, a $29^{\circ} 42^{\prime} \mathrm{S}, 53^{\circ} 49^{\prime} \mathrm{W}$ e a $95 \mathrm{~m}$ de altitude. Nessa área experimental, foram coletados adultos de M. ochroloma e de M. semilaevis. Eles foram transportados ao Laboratório de Entomologia do Departamento de Defesa Fitossanitária da UFSM, colocados em câmara climatizada BOD (temperatura de $25 \pm 2^{\circ} \mathrm{C}$, umidade relativa de $60 \pm 10 \%$ e fotoperíodo de 12 horas) e alimentados com folhas de nabo forrageiro. Os ovos, provenientes das posturas desses insetos, foram mantidos nessa câmara, em placas de Petri de $6 \mathrm{~cm}$ de diâmetro, forradas com papel filtro e umedecidas diariamente até a eclosão das larvas.

Após, em 119 indivíduos de M. ochroloma, foram mensurados os períodos larval (LMO), pupal (PMO) e larval + pupal (LPMO), em dias. Ainda, em 81 indivíduos de M. semilaevis, foram mensurados os períodos larval (LMS), pupal (PMS) e larval + pupal (LPMS). Esses 200 indivíduos foram alimentados com folhas de nabo forrageiro, oriundas de plantas com até seis folhas expandidas. Para cada uma das seis variáveis (LMO, PMO, LPMO, LMS, PMS e LPMS), foram calculadas medidas de tendência central e de variabilidade.

A seguir, para as simulações, foram planejados 118 tamanhos de amostra para as variáveis LMO, PMO, LPMO e 80 tamanhos de amostra para as variáveis LMS, PMS e LPMS. Para as seis variáveis, o tamanho de amostra inicial foi de dois insetos e os demais foram obtidos com incrementos de um inseto até atingir 119 e 81 insetos, respectivamente, para as três variáveis de M. ochroloma (LMO, PMO, LPMO) e para as três variáveis de $\boldsymbol{M}$. semilaevis (LMS, PMS e LPMS). Portanto, para as simulações, foram planejados os seguintes tamanhos de amostra: 2, 3, 4, .., 119 insetos (118 tamanhos) para as variáveis LMO, PMO, LPMO e $2,3,4, \ldots, 81$ insetos (80 tamanhos) para as variáveis LMS, PMS e LPMS.

Após, para cada tamanho de amostra planejado, de cada uma das seis variáveis foram realizadas 10.000 simulações, por meio de reamostragem bootstrap, com reposição. Para cada amostra simulada, foi estimada a média. Assim, para cada tamanho de amostra, de cada variável, foram obtidas 10.000 estimativas de bootstrap da média (FERREIRA, 2009) e determinados os valores mínimo, percentil 2,5\%, média, percentil 97,5\% e máximo. Depois, calculou-se a amplitude do intervalo de confiança de bootstrap de $95 \%$ pela diferença entre o percentil $97,5 \%$ e o percentil $2,5 \%$. Os valores mínimo, percentil 2,5\%, média, percentil 97,5\% e máximo dos 118 tamanhos de amostra $(2,3,4, \ldots, 119$ insetos) das variáveis LMO, PMO, LPMO e dos 80 tamanhos de amostra $(2,3,4, \ldots, 81$ insetos $)$ 
Tabela 1 - Mínimo, máximo, média, mediana, variância, desvio padrão, erro padrão e coeficiente de variação (CV) dos períodos larval, pupal e larval + pupal, em dias, de 119 indivíduos de Microtheca ochroloma e de 81 indivíduos de Microtheca semilaevis.

\begin{tabular}{lllllll}
\hline \multirow{2}{*}{ Estatística } & & & & & & \\
& Larval & Pupal & Larval + Pupal & Larval & Pupal & Larval + Pupal \\
\hline Mínimo & 8 & 3 & 16 & 8 & 5 & 15 \\
Máximo & 17 & 12 & 23 & 16 & 13 & 25 \\
Média $^{(1)}$ & $11,244 \mathrm{a}$ & $6,764 \mathrm{~b}$ & $18,008 \mathrm{a}$ & $9,321 \mathrm{~b}$ & $8,481 \mathrm{a}$ & $17,802 \mathrm{a}$ \\
Mediana & 11,000 & 7,000 & 18,000 & 9,000 & 8,000 & 17,000 \\
Variância & 2,762 & 1,775 & 2,313 & 1,771 & 0,878 & 2,385 \\
Desvio padrão & 1,662 & 1,332 & 1,521 & 1,331 & 0,937 & 1,545 \\
Erro padrão & 0,152 & 0,122 & 0,139 & 0,148 & 0,104 & 0,172 \\
CV (\%) & 14,781 & 19,693 & 8,446 & 14,276 & 11,046 & 8,676 \\
\hline
\end{tabular}

${ }^{(1)}$ Médias de um mesmo período não seguidas de mesma letra diferem entre as espécies pelo teste t com bootstrap de 10.000 simulações em nível de 5\% de probabilidade de erro.

das variáveis LMS, PMS e LPMS foram plotados em gráficos.

A seguir, determinou-se o tamanho de amostra ( $\mathrm{n}=$ número de insetos) para a estimação da média de cada uma das seis variáveis. Para essa determinação, partiu-se do tamanho inicial (dois insetos) e considerou-se como tamanho de amostra o número de insetos a partir do qual a amplitude do intervalo de confiança de bootstrap de $95 \%$ foi menor ou igual a um dia. As análises estatísticas foram realizadas com o auxílio do programa $\mathrm{R}$ ( $\mathrm{R}$ DEVELOPMENT CORE TEAM, 2011) e do aplicativo Office Excel.

A duração do período larval + pupal não diferiu entre as espécies M. ochroloma (18,008 dias) e M. semilaevis (17,802 dias) (Tabela 1). No entanto, a espécie M. ochroloma apresentou maior período larval e menor período pupal em relação à espécie $\boldsymbol{M}$. semilaevis. Esses resultados sugerem que $\boldsymbol{M}$. ochroloma possa causar maior dano às plantas, em função da maior duração do período larval e, consequentemente, maior tempo de alimentação.

Os tamanhos de amostra (número de insetos) para a estimação da média dos períodos larval, pupal e larval + pupal de M. ochroloma, em dias, para uma amplitude do intervalo de confiança de bootstrap de $95 \%$ igual a um dia, foram, respectivamente, 42, $28 \mathrm{e}$ 35 insetos (Figura 1). Para a estimação da média dos períodos larval, pupal e larval + pupal de M. semilaevis, em dias, com intervalo de confiança de bootstrap de 95\% de um dia, os tamanhos de amostra foram, respectivamente, 27,13 e 35 insetos. Não foram encontrados trabalhos na literatura, com metodologia similar a essa, que pudessem ser utilizados para a comparação desses resultados. Com base em outra metodologia de estimação do tamanho de amostra, SILVA \& COSTA (1998) evidenciaram heterogeneidade na distribuição de larvas de Diloboderus abderus Sturm, sendo necessárias 67 e 419 amostras para um nível de erro de baixa precisão (25\%) e de alta precisão (10\%) da média, respectivamente, para oferecer estimativa da população de larvas com uniformidade aceitável.

Em M. ochroloma e M. semilaevis, 42 e 35 insetos, respectivamente, são suficientes para a estimação de duração dos períodos larval, pupal e larval + pupal, com intervalo de confiança de bootstrap de 95\% de um dia.

\section{AGRADECIMENTOS}

Os autores agradecem ao Dr. Luciano de Azevedo Moura, do Departamento de Zoologia, Universidade Federal do Rio Grande do Sul, pela identificação de $\boldsymbol{M}$. semilaevis e de $\boldsymbol{M}$. ochroloma; ao Conselho Nacional de Desenvolvimento Científico e Tecnológico e à Coordenação de Aperfeiçoamento de Pessoal de Nível Superior, pelas bolsas concedidas. 

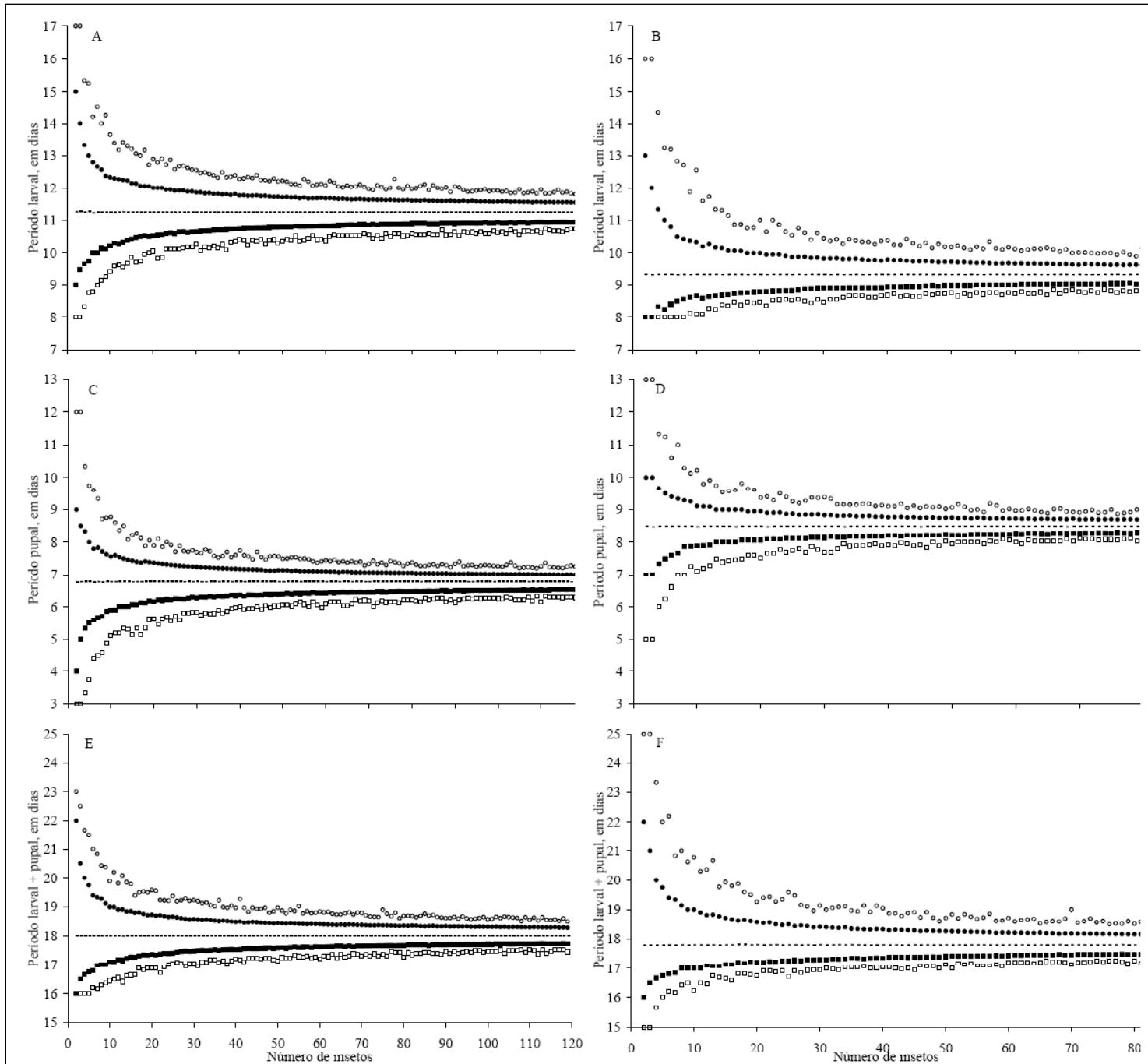

○ Máximo • Percentil 97,5\% - Média - Percentil 2,5\% ロ Mínimo

Figura 1 - Valores mínimo, percentil 2,5\%, média, percentil 97,5\% e máximo das 10.000 estimativas de bootstrap da média de diferentes tamanhos de amostra em número de insetos dos períodos larval, pupal e larval + pupal, em dias, de 119 indivíduos de Microtheca ochroloma (A, C e E) e de 81 indivíduos de Microtheca semilaevis (B, D e F).

\section{REFERÊNCIAS}

FARINHA, L. et al. Occurrence of Microtheca puntigera (Achard) and Microtheca semilaevis Stal in five hosts in the field. Revista Arquivos do Instituto Biológico, v.76, n.3, p.365-371, 2009. Disponível em: <http://www.biologico.sp.gov.br/docs/arq/v76_3/ farinha.pdf $>$. Acesso em: 13 mar. 2011.

FERREIRA, D.F. Estatística básica. 2.ed. Lavras: UFLA, 2009. 664p.

LÚCIO, A.D. et al. Distribuição espacial e tamanho de amostra para o ácaro-do bronzeado da erva-mate. Revista Árvore, v.33, n.1, p.143-150, 2009. Disponível em: <http:// www.scielo.br/scielo.php? script $=$ sci_arttext\&pid=S0100$67622009000100015 \& \operatorname{lng}=$ pt\&nrm=iso $>$. Acesso em: 13 mar. 2011. doi: 10.1590/S0100-67622009000100015.
MANRIQUE, V. et al. Effect of straw mulch on populations of Microtheca ochroloma (Coleoptera: Chrysomelidae) and ground predators in turnip Brassica rapa in Florida. Florida Entomologist, v.93, n.3, p.407-411, 2010.

R DEVELOPMENT CORE TEAM. R: a language and environment for statistical computing. Vienna: $R$ Foundation for Statistical Computing, 2011. Disponível em: http://www.R-project.org. Acesso em: 13 mar. 2011.

SILVA, M.T.B.; COSTA, E.C. Tamanho e número de unidades de amostra de solo para amostragem de larvas de Diloboderus abderus (Sturm) (Coleoptera: Melolonthidae) em plantio direto. Anais da Sociedade Entomológica do Brasil, v.27, n.2, p.193-197, 1998. Disponível em: <http://www.scielo.br/ scielo.php ? script = sci_arttext \& pid=S 0301 $80591998000200004 \& \operatorname{lng}=$ pt\&nrm=iso>. Acesso em: 13 mar. 2011. doi: 10.1590/S0301-80591998000200004

Ciência Rural, v.42, n.1, jan, 2012. 